\title{
Significant Increase of HBV Infection in HIV Infected Patients With Disease Progression in China Based on Two MSM HIV Infected Cohorts
}

\section{Zhiqiang Zhu}

Capital Medical University

Qi Liang

Affiliated Hospital of North Sichuan Medical College

Taiyi Jiang

Capital Medical University

Yanmei Jiao ( $\sigma$ jiaoyanmei@sina.com )

Capital Medical University

Yu Zhang

Capital Medical University

Short report

Keywords: HIV, HBV, Disease progression, Co-infection rate

Posted Date: March 18th, 2021

DOI: https://doi.org/10.21203/rs.3.rs-295395/v1

License: (c) (i) This work is licensed under a Creative Commons Attribution 4.0 International License.

Read Full License 
Significant increase of HBV infection in HIV infected patients with disease progression in China based on two MSM HIV infected cohorts

Zhiqiang Zhu ${ }^{1 \#}$, Qi Liang ${ }^{2 \#}$, Taiyi Jiang ${ }^{3}$, Yanmei Jiao ${ }^{3}$, Yu Zhang ${ }^{1 *}$.

1, Department of Urology, Beijing You'an hospital, Capital Medical University, Beijing 100069, China

2, Laboratory department, Affiliated Hospital of North Sichuan Medical College

3, Center for Infectious Diseases, Beijing YouAn Hospital, Capital Medical University, Beijing 100069, China

*Corresponding author: Yu Zhang email: zhangyud@hotmail.com

\# Those author contribute equal to this work. 


\begin{abstract}
The date about the condition of HBV co infection with the disease progress of HIV is limited. To investigate whether the incidence of HBV co-infection is significantly higher in HIV patients with disease progression in China, we compared rates of HBV co-infection in HIV patients based on an acute and a chronic HIV infected cohort. Significance was assessed with Chi-square. HBV infection is diagnosed by the presence of hepatitis B surface antigen. The HBsAg positive rate increased from $6.18 \%$ in acute HIV infection to $11.44 \%$ in chronic HIV infection. Thirty-four acute HIV patients had been tested for HBV in their chronic phase, four of them had HBV -specific antigens and/or specific antibodies changes. The number of Hepatitis B virus-specific antibodies decreased from acute phase to chronic phase in four patients and two patients' HBsAg changed from negative to positive. There is an increased prevalence of HBV infection in HIV patients with the disease progression in China. Key words: HIV; HBV; Disease progression; Co-infection rate.
\end{abstract}




\section{Introduction}

The issue of viral co-infection among human immunodeficiency virus (HIV) /acquired immunodeficiency syndrome (AIDS) patients represents a growing public health concern worldwide. HIV and hepatitis B virus (HBV) have same routes of transmission (1), and HIV/HBV coinfection is associated with liver-related disease and morbidity and mortality rates (2). HIV-infected persons are less likely to clear acute HBV infection spontaneously when their immune system is significantly compromised (3).

China has the highest prevalence of chronic HBV with carrier rates of more than $7 \%{ }^{(4)}$. HBV infection in China has remained a public health issue and a major cause of mortality. So far, there are no defined studies to show that in what way HIV infection will facilitate HBV infection in China. It is imperative to obtain evidence about whether and the extent the progression of HIV infection can facilitate the HBV co-infection in China.

Since 2016, China has implemented a strategy of treatment immediately after HIV infection is detected. In this study, two cohorts of HIV infection were established in Youan hospital before $2016^{(5)}$, one was the acute phase of HIV infection, the other was the chronic phase of HIV infection. Without treatment, we observed the situation of HBV co infection with the progress of HIV disease. The purpose of this study was to investigate if HBV co-infection is significantly increased in HIV patients with disease progression in China.

\section{Materials and Methods}




\subsection{Study population}

The data were collected from clinic-based MSM (men who have sex with men) cohorts in Beijing Youan Hospital from June 2006 to August 2015. Both acute and chronic HIV infection cohorts were incorporated in this study. The characteristic of patients are shown in Table 1. This study was approved by the Ethical Committee at Beijing Youan Hospital (YAH200605010) and written informed consent was obtained from all participants.

For acute HIV cohort, there were 372 consenting patients who were recently infected (from Fiebig stage I to Fiebig stage V (4)) with HIV. All patients were from a cohort of HIV-negative high-risk MSM who were screened every 2 months for HIV infection in Beijing You-an Hospita (5). CD4, viral load, HBV-specific antigens and specific antibodies were detected after identification of HIV infection.

For chronic cohort, there were 306 HIV-infected individuals with diagnosis of HIV infection for more than 6 months for their first visit. CD4, viral load, HBV-specific antigens and specific antibodies were detected for the first visit. All patients have not been on antiretroviral treatment.

\subsection{Detection of HBV Infection}

HBV-specific antigens and specific antibodies in patient plasma were detected in the clinical laboratory at Youan hospital using the Elecsys ${ }^{\circledR}$ HBsAg Immunoassay (Roche diagnostics GmbH, Mannheim, Germany), the immunoassay analyzer cobase 411 (Roche diagnostics GmbH, Mannheim, Germany), or specific ELISA test kits (PRECHEK Bio, Anaheim, USA), respectively, according to manufacturer's instructions.

HBV infection is diagnosed by the presence of hepatitis B surface antigen (HBsAg), which is 
indicative of acute or chronic infection (6).

\subsection{Liver Function Tests}

Alanine aminotransferase (ALT) and aspartate aminotransferase (AST) levels were determined in patient plasma by UV-LDH method test kit (Fortress Diagnostics Limited, United Kingdom).

\subsection{Markers of HIV disease progression}

Absolute blood CD4+ T-cell counts were measured using a FACSCalibur flow cytometer.

Viral load was measured by the Amplicor HIV monitor ultrasensitive method with a detection limit of 40 copies/mL of plasma.

\subsection{Statistical analyses}

All statistical analyses were performed using SPSS 23.0 (Chicago, IL, USA). Two-sided $p<0.05$ was accepted as statistically significant. The Chi-square test for categorical variables were used for comparison between two groups.

\section{Results}

The data were collected from outpatient clinic-based MSM (men who have sex with men) cohorts managed in Beijing Youan Hospital from June 2006 to August 2012. In the acute cohort, 23 out of 372 cases were $\mathrm{HBsAg}$ positive; HBsAg positive rate was $6.18 \%$. In chronic cohort, were a total of 306 chronic HIV infected patients, 35 out of 306 patients were HBsAg positive, HBsAg positive rate was $11.44 \%$. The rate of $\mathrm{HBV}$ co infection in chronic HIV infection was significantly higher than that in acute stage $(p=0.015$, Figure 1). The CD4 count decreased from average 445 in acute infection to 358 in chronic 
infection $(\mathrm{p}<0.001)$ (Table 1).

In total, 34 acute patients had detected HBV in the chronic phase, 4 of them had HBV -specific antigens and (or) specific antibodies changes (Table 2). CD4 counts decreased and viral load increased in these four cases from acute phase to chronic phase. The number of Hepatitis B virus-specific antibodies decreased from acute phase to chronic phase in four patients and two patients' HBsAg changed from negative to positive.

\section{Discussion}

We found HBV co-infection significantly increased from acute HIV infection to chronic HIV infection in China. Two cases in acute cohort have been detected HBV-specific antigens in chronic phase compared with HBV specific antibodies in acute HIV infection. All evidence suggested that the rate of HBV infection increased with the progression of HIV infection. Data presented in this report obtained from one of major clinic in Beijing. Most HBV infection is acquired during the perinatal period or early in childhood in high prevalence areas. In this study, we observed obvious increase in the rate of HBV infection with the disease progression and immune system damage in adult HIV patients. cccDNA has been shown to persist even in the liver of patients with successful cellular and humoral control of the infection (7). It suggests the possibility that HBV virus comes from cccDNA in the liver tissue. Other studies have shown that reactivation of HBV after undergoing anticancer therapy or rituximab-containing treatment $(8,9)$.

Given that HIV/HBV co-infection results in higher morbidity and mortality of liver disease compared to HBV mono-infection, greater attention should be paid to control HBV infections 
in HIV patients in China. As the immune restoration by HAART treatment can improve HBV modulation, preventive regimen should be taken into consideration for immune compromised patients with HIV infection in China.

\section{Abbreviations}

HIV: human immunodeficiency virus; AIDS: acquired immunodeficiency syndrome; HBV: hepatitis B virus; MSM: men who have sex with men; HBsAg: hepatitis B surface antigen;

ALT: Alanine aminotransferase; AST: Aspartate aminotransferase.

\section{Declarations}

\section{Ethics approval and consent to participate}

This study was approved by the Ethical Committee at Beijing Youan Hospital (YAH200605010) and written informed consent was obtained from all participants.

\section{Consent for publication}

Not applicable.

\section{Availability of data and materials}

All relevant data are within the manuscript. The datasets collected and/or analyzed during the current study are available on request from the author.

\section{Conflict of interest statement}

The authors declare no conflict of interest.

\section{Authors' contributions}

ZZ has made substantial contributions to the conception; YZ design of the work; QL has done the laboratory; TJ interpretation of data; YJ have drafted the work or substantively revised it. 


\section{Funding statement}

This work was supported by the National $13^{\text {th }}$ Five-Year Grand Program on Key Infectious

Disease Control (2018ZX10301407-005-001).

\section{Acknowledgments}

We would like to acknowledge Beijing Key Laboratory of AIDS for provision of all laboratory materials.

\section{References}

1. Kim AY. Hepatitis B Virus and HIV Coinfection: Fibrosis, Fat, and Future Directions. American Journal of Gastroenterology. 2019;114:710-2.

2. Thornton AC, Jose $S$, Bhagani $S$, et al. Hepatitis $B$, hepatitis $C$, and mortality among HIV-positive individuals. Aids. 2017;31:2525-32.

3. Sarmati L, Malagnino V. HBV Infection in HIV-Driven Immune Suppression. Viruses-Basel. 2019;11.

4. Wang FS1, Fan JG, Zhang Z, Gao B, Wang HY. The global burden of liver disease: the major impact of China. Hepatology. 2014 Dec;60(6):2099-108. doi: 10.1002/hep.27406. Epub 2014 Oct 29.

5. Huang X1, Chen H, Li W, Li H, Jin X, Perelson AS, Fox Z, Zhang T, Xu X, Wu H. Precise determination of time to reach viral load set point after acute HIV-1 infection. J Acquir Immune Defic Syndr. 2012 Dec 1;61(4):448-54. doi: 10.1097/QAI.0b013e31827146e0.

6. Fiebig EW, Wright DJ, Rawal BD, et al. Dynamics of HIV viremia and antibody seroconversion in plasma donors: implications for diagnosis and staging of primary HIV infection. Aids. 2003;17:1871-9.

7. Jiao Y, Zhang T, Wang R, et al. Plasma IP-10 is associated with rapid disease progression in early HIV-1 infection. Viral immunology. 2012;25:333-7.

8. Ujiie I, Ujiie $\mathrm{H}$, Yoshimoto $\mathrm{N}$, et al. Prevalence of infectious diseases in patients with autoimmune blistering diseases. The Journal of dermatology. 2020.

9. Werle-Lapostolle B, Bowden S, Locarnini S, et al. Persistence of cccDNA during the natural history of chronic hepatitis B and decline during adefovir dipivoxil therapy. Gastroenterology. 2004;126:1750-8.

10. Yeo W, Chan TC, Leung NW, et al. Hepatitis B virus reactivation in lymphoma patients with prior resolved hepatitis B undergoing anticancer therapy with or without rituximab. Journal of clinical oncology : official journal of the American Society of Clinical Oncology. 2009;27:605-11.

11. Pei SN, Chen $\mathrm{CH}$, Lee CM, et al. Reactivation of hepatitis $\mathrm{B}$ virus following rituximab-based regimens: a serious complication in both $\mathrm{HBsAg}$-positive and HBsAg-negative patients. Annals of hematology.

2010;89:255-62. 


\section{Figures}

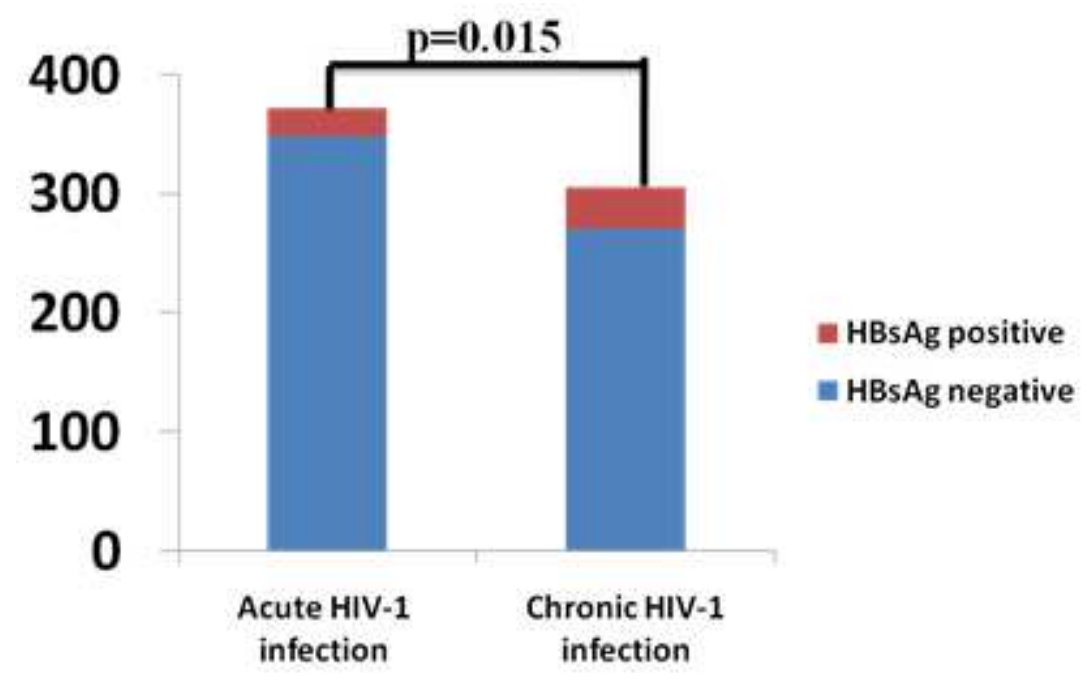

Figure 1

In chronic cohort, were a total of 306 chronic HIV infected patients, 35 out of 306 patients were HBsAg positive, HBsAg positive rate was $11.44 \%$. The rate of HBV co infection in chronic HIV infection was significantly higher than that in acute stage $(p=0.015$, Figure 1$)$.

\section{Supplementary Files}

This is a list of supplementary files associated with this preprint. Click to download.

- graphicalabstract.docx 